\title{
PARTÍCULAS VERBAIS E ASPECTO NAS COMPLEX PARTICLE CONSTRUCTIONS
}

\author{
Andrea Knöpfle
}

\begin{abstract}
Resumo
O objetivo deste trabalho é abordar a contribuição para a estrutura de evento da partícula verbal em complex particle constructions. O recorte será um subgrupo destas construções que apresenta um predicado secundário com leitura de estado resultante sobre um sintagma acusativo juntamente com a presença de uma partícula verbal, conforme se observam nos exemplos seguintes: John grilled the onions up dry e The farmer painted the barn up red. A argumentação é de que a contribuição da partícula é de natureza aspectual, mais especificamente, com o sentido de completude e ou totalidade, e não telicidade propriamente.

Palavras-chave: Partícula Verbal; Aspecto; Resultado; Complex Particle Constructions
\end{abstract}

\section{VERB PARTICLES AND ASPECT IN COMPLEX PARTICLE CONSTRUCTIONS}

\begin{abstract}
The goal of this paper is to investigate the verb particle's contribution for the event structure in complex particle constructions. The focus will be on a subgroup of constructions that have a secondary predicate denoting a resultant state over an accusative phrase alongside with a verb particle, as we can see in the following examples: John grilled the onions up dry and The farmer painted the barn up red. The argumentation supports the view that the particle contribution is aspectual in its nature, specifically representing a sense of completeness and/or totality, and not telicity proper.

Key-words: Verb Particle; Aspect; Result; Complex particle constructions
\end{abstract}

\footnotetext{
*Doutora pelo Programa de Pós-Graduação em Letras da Universidade Federal do Paraná (UFPR), área de concentração em Estudos Linguísticos, teoria e análise gramatical. Mestre em Letras, Estudos Linguísticos, pela UFPR. De 2015 a 2019, foi professora adjunta do Departamento de Letras da Universidade Federal de Pernambuco. Em 2015, foi professora colaboradora do Departamento de Estudos da Linguagem da Universidade Estadual de Ponta Grossa (UEPG).Em 2014, foi professora substituta do Departamento de Linguística da UFPR. E-mail: deaknoepfle@gmail.com. ORCID: https://orcid.org/0000-0002-6656-2095.
} 


\section{Introdução}

Partículas combinadas a verbos têm despertado grande interesse na literatura, desde a tradição gramatical até as pesquisas mais recentes em linguística (ver, por exemplo, BOLINGER, 1971;FRASER, 1976; LINDNER, 1981;DEN DIKKEN,1995;CAPELLE, 2005; e, no Brasil, MARCELINO, 2007, 2014; BARBOSA, 2012;KNÖPFLE, 2014, 2018;ENDRES, 2013; PIEROZAN, 2016).Neste artigo, o foco de estudo é o inglês, mas esse tipo de fenômeno ocorre, em maior ou menor grau, em outras línguas germânicas (cujos exemplos serão eventualmente também apresentados). As construções verbo-partícula também são chamadas de frasal verbs ${ }^{1}$.

Tipicamente, partículas (tomadas também como preposições)se combinam a verbos e agregam desde um significado mais composicional (lexical, aspectual), como em (1a), até idiomático, como em (1b).

(1) a. We broke up the crackers into smaller pieces.

b. We broke up last week after a fight.

A contribuição da partícula, em negrito, no primeiro exemplo, pode ser tomada como sendo aspectual: desde telicidade até a ideia de completude. Já no segundo exemplo, o sentido (combinado com o verbo) é de algum término (de um relacionamento). Interessa, para este trabalho, o sentido composicional (e especificamente aspectual) que as partículas agregam na estrutura de evento. Vale notar que o estatuto aspectual das partículas (a exemplo de up) enquanto telos do evento também é controverso, sendo questionado até que ponto a partícula encerraria o evento.

A questão aspectual será retomada neste trabalho em construções verbo -partícula, mais especificamente, em complex particle constructions ${ }^{2}$ (ver DEN DIKKEN, 1995), em que a partícula ocorre juntamente com um predicado secundário, a exemplo de (2):

(2) a. John grilled the onions up $\underline{\text { dry. }}$.
b. The farmer painted the barn up red.

Os exemplos em (2) trazem um predicado secundário representado pelos APs, grifados, juntamente com a partícula verbal, em negrito. Construções desse tipo, sem a partícula, são conhecidas na literatura como pseudoresultativas e resultativas, respectivamente(CARRIER; RANDALL, 1992;LEVIN; RAPPAPORT, 1995;HOEKSTRA, 1988, 1992;KRATZER, 2005; LEVINSON, 2007, 2010; inter alia).Em termos aspectuais, o predicado secundário confere telos ao evento: a denotação do sintagma adjetival (AP) representa o resultado do evento, acionado pelo verbo, sobre o sintagma determinante acusativo $\left(\mathrm{DP}_{\mathrm{ACC}}\right)$.

Neste ponto, podemos introduzir a questão central do presente artigo: se a partícula é de natureza aspectual, então qual seria sua contribuição na estrutura 
de evento? A argumentação é de que tal contribuição, de natureza aspectual, encerra completude, e não telicidade propriamente.

Assim, as estruturas em (2a-b) têm um sentido equivalente a se entender, respectivamente, que "as cebolas foram grelhadas de forma que ficaram completamente/totalmente secas" e "houve uma ação de pintar que teve como resultado o celeiro ficar todo/completamente vermelho". Na argumentação defendida aqui, portanto, o predicado secundário (grifado nos dados em (2) e na paráfrase acima) indica a telicidade do evento; e a partícula (em negrito nos dados em (2) e na paráfrase acima) indica totalidade e/ou completude.

Com o objetivo de esclarecer essa linha argumentativa, o artigo está estruturado da seguinte maneira: na primeira seção, são tecidas algumas considerações sobre aspecto as quais são relevantes para este artigo. Na seção 2, retomam-se as partículas verbais e a complexidade de sua categorização gramatical, com exemplificações acerca dos sentidos para fundamentar a discussão. Na terceira seção, são detalhadas as complex particle constructions, com o objetivo de se alcançar o subgrupo destas partículas que é foco deste trabalho: partículas em construções resultativas e pseudoresultativas. Na seção 4, essas construções são apresentadas em maior detalhe, sobretudo no que se refere à aspectualidade. A quinta seção encerra o debate com algumas considerações.

\section{Aspecto}

Esta breve seção tem como objetivo colocar algumas assunções básicas sobre aspecto que se farão relevantes no decorrer do artigo. Assim, toma-se aspecto como se referindo ao aspecto interno do evento (inner aspect), no sentido de aspecto lexical (relacionado às classes aspectuais ou Aktionsarten e caracterizado por propriedades lexicais), em que também se encontra a oposição de télico e atélico. Essas noções aspectuais não estão limitadas ao léxico: segundo Vendler (1967), fatores como a presença ou não de um objeto verbal (um DP acusativo, digamos) também pode fazer parte da interpretação lexical.

Vendler (1967) divide os eventos (ou eventualidades) em quatro classes aspectuais: atividades, estados, accomplishments e achievements, distinguindo-as em termos de instantes e intervalo de tempo. Rothstein (2004) define didaticamente essas classes: os estados são caracterizados como eventualidades totalmente homogêneas até o seu menor instante, onde cada subparte de um estado é o próprio estado; as atividades são eventualidades dinâmicas e homogêneas até intervalos mínimos, em que os intervalos mínimos têm um determinado tamanho (em outros termos, há eventos mínimos dentro da atividade, mas não é qualquer instante dentro da atividade que caracteriza a atividade em si); achievements são mudanças de estado que ocorrem instantaneamente; e accomplishments são eventualidades complexas com certa duração, que apresentam um ponto de culminação. Vejamos exemplos das quatro classes (estados, atividades, accomplishments e achievements, respectivamente): 
(3) a. Os alunos sabem matemática.

b. As crianças nadam bem.

c. A Teresa comeu duas maçãs.

d. A Renata perdeu a caneta. (Wachowicz e Foltran, 2007, p. 3).

Para refinar as classes aspectuais vendlerianas, Rothstein (2004) coloca os traços [ \pm estágio] e [ \pm télico]. As eventualidades achievement e os estados não possuem estágios; as achievements são instantâneas, o que impossibilita a distinção de estágios nesse momento instantâneo; os estados, por sua vez, mesmo sendo potencialmente longos, não são dinâmicos e cada momento de um estado é o mesmo durante toda a eventualidade, impossibilitando igualmente o reconhecimento de estágios. Diferentemente, as atividades são caracterizadas como [+ estágio] e [- télico].

Segundo a autora, a característica [- télico] em atividades se deve ao fato de que atividades são cumulativas, ou seja, a soma de duas atividades continua sendo uma só atividade, isto é, a soma forma um novo evento singular. Como atividades são homogêneas até eventos mínimos (e não instantes mínimos, como em estados), elas possuem a característica [+ estágio], em que os estágios são reconhecidos nesses eventos mínimos em que há algum movimento ou mudança de estado. Quanto às eventualidades accomplishments, essas são caracterizadas com os traços [+ estágio] e [+ télico], observando sua característica de atividade culminada.

De posse dessas definições básicas, a próxima seção discorre sobre as partículas verbais, inclusive abordando a discussão acerca das propriedades aspectuais desses elementos dentro da estrutura de evento.

\section{Partículas verbais}

Os sentidos e a categorização gramatical das partículas verbais são temas complexos: são também categorizadas como preposições ou mesmo advérbios (ver, por exemplo, BOLINGER, 1971;FRASER, 1976;LINDNER, 1981; CAPELLE, 2005), e suas combinações com um núcleo verbal estabelecem significados dos mais variados tipos, desde níveis mais composicionais de sentidos mais prototípicos até níveis mais idiomáticos.

Não é objetivo desta seção uma descrição abrangente dos usos e sentidos das partículas - o que fugiria dos propósitos deste artigo. A ideia, em primeiro lugar, é buscar, por meio da ilustração do sentido em alguns exemplos, pistas de contribuições aspectuais das partículas na estrutura de evento.Na sequência, a seção aponta a discussão acercada categorização gramatical (e, em alguma medida, teórica) para as partículas.

\subsection{Sobre partículas e aspecto verbal}

Em termos de sentido, Rudzka-Ostyn (2003) propõe esquemas imagéticos (dentro de uma abordagem semântico-cognitiva, com base em Lakoff e Johnson 
(1980)) para ilustrar a questão semântica referente às partículas up e down. A primeira partícula é tida como a mais utilizada em língua inglesa, segundo Rudzka -Ostyn (2003). São 6 os sentidos para up descritos pela autora, conforme explica Pierozan (2016) em comparação com a língua portuguesa. Interessam-nos aqui especialmente os sentidos descritos em (4) e exemplificados respectivamente em (5):

(4) a. up: uma posição superior ou mover em direção a um lugar mais alto

b. up: cobrir uma área completamente/atingir o limite máximo

(5) a. Put the bottle up on the shelf.

b. Cut up the meat! ${ }^{3}$

Os sentidos descritos para down vão na direção contrária aos descritos para up: posição inferior ou movimento para um lugar mais baixo; atingir um objetivo, realização, completude, limite em uma escala para baixo. Juntamente com up e down, as partículas out e off estão entre as mais frequentes, segundo Rudzka -Ostyn (2003).

Endres (2013) se concentra na descrição das partículas segundo suas propriedades aspectuais. Segundo a autora, as partículas que podem adicionar continuatividade para as situações são on, along e away, ao passo que as partículas up, down, out, off, through, over e away podem adicionar telicidade às situações. Abaixo, os grupos são exemplificados, com as respectivas paráfrases indicando a aspectualidade (Endres, 2013, p.16):

(6) a. The place was filling up.

a. The place was filling completely.

b. Mike stood up and walked on after he fell.

b. Mike stood up and continued to walk after he fell.

Basicamente, a autora assume que partículas télicas se unem a um verbo atélico (uma atividade, por exemplo), transformando a eventualidade em accomplishment, portanto conferindo telicidade ao evento. Os testes sugeridos são os encontrados tradicionalmente na literatura (como em DOWTY, 1979) para verbos simples de atividade: a adição de um sintagma determinante compara-se à adição da partícula para a mudança de Aktionsart, como vemos no exemplo:
(7) a. Annie was drawing.
b. Annie was drawing a circle.
c. Annie was cleaning the house. $\rightarrow$
d. Annie was cleaning up the house.

A partícula down é comparada à up como telos/completude do evento, com significado adicional semelhante a algo como "down to the ground", "down to destruction" ou "down to the feet", como nos exemplos da autora (p.37): 
(8) a. You have to cut down the sugar from your diet.

b. Ted was knocked down by Barney.

A partícula off também traz o sentido de completude, segundo a autora, em que à telicidade se une um significado adicional de ênfase à terminação, à completude. São exemplos da autora (p. 39):

(9) a. The movie ended because they had killed off the characters.

b. Alison is relieved because she has paid off her credit card bill.

A partícula télica out pode, segundo Endres (2013, p.40), conferir tanto telicidade 'pura' quanto o sentido de 'desaparecer completamente':

(10) a. Please, I'm begging you to just hear me out.

b. When Teresa got home, sunshine was already fading out.

Concluindo a exemplificação, a partícula away apresenta, juntamente com sentido de telos, o sentido de 'desaparecimento':

(11) a. The conversation slowly died away.

b. His ex-wife has spent his money away. ${ }^{4}$

Capelle (2005) discute e, em certa medida, questiona a assunção acerca da telicidade das partículas verbais, a exemplo de up, com base nos testes tradicionais de telicidade. A questão é se a partícula verbal altera de fato a estrutura do evento em termos de lhe conferir telicidade. O exemplo clássico, usado a favor da telicidade da partícula, seria algo semelhante a (12), abaixo, em que a adição da partícula confere telicidade ao evento.

(12) a. They drank the beer for/ in 5 minutes.

b. They drank up the beer ${ }^{\star}$ for/ in 5 minutes.

Apesar de não se tratar do uso mais produtivo da partícula aspectual, o autor argumenta que, nesse caso, não se trata de telicidade em si, mas de completude. Capelle defende o uso de partículas prototipicamente direcionais (away, back, down, off, on, through, out, up, etc.) com sentido de desdobramento temporal, porém conclui que não se pode generalizar para um telos.

As noções de completude e telicidade estão intimamente relacionadas, tanto que a primeira pressupõe a segunda. Ainda assim, Capelle apresenta evidências empíricas a favor da separação entre essas duas noções em alguns casos, quando se trata da contribuição da partícula aspectual para a eventualidade.

Para o exemplo em questão, trata-se de um uso com leitura resultativa: nesse caso, a partícula indica um predicado resultativo subespecificado, parafraseado em algo como consume your beer by drinking it, em que up assume um 
significado de consumido ou exaurido (similarmente a the time is up). Seria o caso, então, de estarmos lidando com dois padrões de up, exemplificado pela ambiguidade de chew the meat up, que teria uma leitura resultativa (cause the meat to get in a chewed state, i.e., make it squishy), e um sentido de exaustão (consume the meat by chewing it, i.e., eat it). Somente a última leitura trata de uma versão télica do verbo chew.

\subsection{Sobre a categorização gramatical das partículas verbais}

As combinações verbo-partícula, como já indicado na introdução, também são conhecidas como frasal verbs. Não é raro se referir às partículas, nessas construções, como preposições e até mesmo advérbios. Antes de uma simples questão terminológica, trata-se de uma categorização gramatical que envolve distribuição morfossintática e semântica. Dada a complexidade do tema e o recorte do trabalho, o objetivo aqui é tão somente apontar a discussão, referida com base em dois autores: den Dikken (1995) e Capelle (2005).

Den Dikken (1995) estuda (particularmente a distribuição das) partículas verbais como evidência empírica para a sintaxe das complex particle constructions, em que a partícula faz parte da construção (assunto da próxima seção). Sobre a categorização gramatical da partícula, o autor defende que elas são elementos de natureza preposicional (com base em EMONDS, 1972, 1976), em que as partículas são tomadas como preposições intransitivas). Uma vez intransitivas, não tomam mais de um argumento. ${ }^{5}$

Sintaticamente, são apontadas duas principais possibilidades: estudos que partem da premissa de que a partícula forma uma unidade lexical com o verbo, e estudos que tomam a partícula enquanto um núcleo sintático independente (ver a discussão em EMONDS, 1972, 1976). Baseando-se no último grupo, a partícula é então analisada como sendo um núcleo Ergativo (de uma Small Clause) pertencente à categoria gramatical $\mathrm{P}$ (reposição).

O interesse do autor não é tanto no sentido em si, mas sim em acomodar as várias possibilidades de distribuição sintática das partículas. Independentemente de seu rótulo gramatical, relevante é tomá-la como núcleo sintático autônomo para dar conta de sua distribuição. Assim, pode-se evitar, por exemplo, mecanismos como excorporação que seriam necessários caso ela fizesse parte de uma unidade lexical com o verbo.

Capelle (2005) reconhece a vertente que trata as partículas como elementos preposicionais, porém argumenta no sentido de que elas fazem parte de uma classe gramatical à parte. Para tanto, o autor defende três pontos: partículas e sintagmas preposicionais têm comportamento sintático distinto; partículas não podem ser tomadas como sintagmas preposicionais reduzidos e partículas não possuem o mesmo significado que as preposições a elas relacionadas. O objetivo de Capelle é diferenciar partículas de preposições, porém, assumindo que as similaridades podem ser entendidas se as partículas forem tomadas como uma espécie de preposição intransitiva, diferentemente de full PPs (na terminologia 
do autor). Capelle conclui que a terminologia 'partícula' é mais econômica que 'preposição intransitiva'.

De uma forma ou de outra, a questão acerca da categorização gramatical das partículas (enquanto elementos preposicionais ou classe gramatical independente) permanece assunto aberto para debate e investigação.

\section{Complex particle constructions}

\subsection{As complex particle constructions em linhas gerais}

A sintaxe de construções verbo-partícula, construções triádicas e construções causativas é estudada em den Dikken (1995), que se refere a esses tipos de estruturas como complex particle constructions. A proposta do autor é uma abordagem unificada, estruturada em relações de sujeito e predicado sem tempo, chamadas de Small Clauses (SC). Nesse contexto, as partículas entram na estrutura como um indicativo empírico para a proposta de descrição estrutural. Trata-se de construções de objeto duplo, causativas e (pseudo)resultativas. Vejamos os exemplos (DEN DIKKEN, 1995, p.36):

(13) a. They made John out (to be) a liar.

b. They turned out (to be) intelligent.

c. They painted the barn up red.

d. They put the books down on the shelf.

e. They sent a schedule out to the stockholders.

Nas complex particle constructions há a presença de um verbo, uma partícula e um predicado adicional/secundário. Nos exemplos acima, a partícula é dada em negrito e o predicado adicional, em itálico. ${ }^{6}$ Den Dikken analisa as partículas como preposições funcionais (seguindo EMONDS, 1985 e KAYNE, 1985) que, em termos semânticos, são similares a verbos auxiliares por não atribuírem um papel temático ao argumento externo. Quanto à distribuição sintática, pertencem à categoria $\mathrm{P}$ (reposicional) e são núcleo da SC complemento do verbo. Essa SC complemento, nucleada pela partícula, toma uma outra SC como complemento que encerra uma relação de sujeito e predicado (entre 'the barn' e 'red', como no dado (13c), sendo a teoria para a análise baseada em Barriers (CHOMSKY, 1986)). Esquematicamente, temos:

(14) a. They painted the doorupred.

b. $\mathrm{V}\left[{ }^{\mathrm{SC} 1}\right.$ up [ ${ }^{\mathrm{SC} 2}$ [the door] [red]]

c. They painted $<^{*}$ up $>$ the door $<$ up $>$ red.

O núcleo da SC1 é preenchido pela partícula ergativa e, portanto, não atribuidora de argumento externo, fazendo de seu Spec um possível landing site para movimento. Assim, a ordem de superfície em (14a) é derivada por meio do mo- 
vimento obrigatório do DP [the barn] para checagem de Caso (em uma configuração ECM). Esse movimento obrigatório também dá conta da restrição da distribuição da partícula, como em (14c).

Den Dikken segue na análise das outras construções exemplificadas em (13) e suas respectivas restrições na distribuição das partículas. $\mathrm{O}$ recorte deste artigo, conforme sinalizado na introdução, é um subgrupo das complex particle constructions: as construções resultativas e pseudoresultativas em que há adição de partícula (argumentavelmente de natureza aspectual). Esse tipo de construção está exemplificado em (2), (13c) e (14a) e será melhor descrito na próxima subseção.

\subsection{Construções (pseudo)resultativas}

Tomemos, então, construções (pseudo)resultativas com adição de partícula como um subgrupo das complex particle constructions. Para tanto, é relevante abordar, ainda que brevemente, as construções (pseudo)resultativas em si, independentemente da adição de partícula.

Existeumvastodebatenaliteraturatécnicaacercadessasestruturas, sendorepresentativosostrabalhosdeCarriereRandall(1992), LevineRappaport(1995), Hoekstra (1988, 1992), Geuder (2000), Kratzer (2005), Levinson (2007, 2010), inter alia. Nas construções resultativas, produtivas em línguas ocidentais germânicas (KNÖPFLE, 2014), o verbo principal (V) denota uma ação (cujo agente é expresso pelo sujeito $\left.\left(\mathrm{DP}_{\mathrm{NOM}^{\prime}}\right)\right)$, sendo o estado resultante da ação denotado pela combinação de um sintagma adjetival (AP) ou ainda preposicional (PP) (o sintagma resultativo)e um DP Acusativo $\left(\mathrm{DP}_{\mathrm{ACC}}\right)$. Vejamos alguns exemplos do inglês, holandês e alemão:

(15) a. He painted the house yellow. (inglês)

Ele pintou a casa amarelo

'Ele pintou a casa, que ficou amarela.'

b. Hij kocht de winkel leeg. (holandês)

Ele comprou o mercado vazio

'Ele comprou (tanto/de forma tal) que o mercado ficou vazio.'

c. Der Patient hat das Leintuch schmutzig geblutet.(alemão)

O paciente teve o lençol sujo sangrado

'O paciente sujou o lençol, ao sangrar sobre ele.'

d. Hijschreeuwdezijn keel rauw. (holandês)

d. He screamed his throat sore. (inglês)

Ele gritou sua garganta machucado/inflamado.

'Ele gritou (tanto/de forma tal) que sua garganta ficou machucada/

inflamada.' 
O que resta de mais intrigante nas resultativas tem relação com a estrutura argumental: o $\mathrm{DP}_{\mathrm{ACC}}$, necessariamente afetado pelo verbo (affected object, nos termos de HOESKTRA, 1988), não precisa ser argumento de V (ou semanticamente selecionado por $\mathrm{V})^{7}$. Nos exemplos acima, em (c-d), $\mathrm{V}$ é intransitivo, mas os resultados das ações de 'sangrar' e 'gritar' têm como resultado 'o lençol sujo' e 'a garganta machucada', respectivamente. No exemplo em (b), apesar de o verbo poder ter uma leitura transitiva, 'o mercado' não é objeto do verbo, mas resulta em 'vazio' por meio da ação verbal. O exemplo em (a) tem um verbo transitivo, e o resultado da ação tem como resultado 'a casa amarela.' ${ }^{8}$ Podemos também obter dados ambíguos entre uma leitura transitiva ou intransitiva:

(16) a. Hans hat seine Hand kaputt gehämmert. (alemão)

Hans teve sua mão machucada martelado

a. Hans hammered his hand sore. ${ }^{9}$ (inglês)

leitura 1: 'Hans martelou sua (própria) mão, que ficou machucada por conta disso.' leitura transitiva

leitura 2: 'Hans martelou algo, e porque ele fez isso (durante horas), sua mão ficou machucada.' leitura intransitiva

A generalização que se segue na literatura mencionada é que, independentemente da descrição estrutural, (i) o objeto afetado pelo verbo pré-existe à ação verbal; (ii) a supressão do sintagma resultativo gera dados agramaticais com verbos intransitivos; (iii) é possível a presença de apenas um sintagma resultativo em cada construção, e (iv) o adjetivo (ou sintagma resultativo, de uma forma geral) sempre predica (atribui propriedade) do $\mathrm{DP}_{\mathrm{ACC}}$, e nunca do sujeito da sentença ${ }^{10}$.

As construções pseudoresultativas (também chamadas de resultativas adverbiais) são muito semelhantes em superfície às resultativas. As distinções e propostas de análise são encontradas nos trabalhos de Parsons (1990), Geuder (2000), Kratzer (2005) e Levinson (2007, 2010), inter alia. Seguem exemplos:

(17) a. I opened the door wide.

Eu abri a porta amplo

'Eu abri a porta escancarada/bem aberta.'

b. I shut the door tight. ${ }^{11}$

Eu fechei a porta apertado

'Eu fechei a porta bem fechada.'

c. He chopped the parsley fine.

Ele picou a salsa fino

'Ele picou a salsa fininha.' 
d. He cut the hair short

Ele cortou o cabelo curto

'Ele cortou o cabelo curto.'

e. She sewed the skirttight.

Ela costurou a saia justo

'Ela costurou a saia justa.'

Parsons (1990) nota que a principal diferença entre resultativas e pseudoresultativas é que, nas últimas, os APs não denotam o estado resultante em si, mas atuam como uma espécie de modificador de estado resultante, já denotado em [V $\mathrm{DP}_{\mathrm{ACC}}$. Assim, o $\mathrm{DP}_{\mathrm{ACC}}$ precisa necessariamente ser argumento de V. Os trabalhos subsequentes acerca de pseudoresultativas (a exemplo de Geuder, Kratzer e Levinson) seguem refinando nuances semânticas e distinções empíricas dentro do grupo pseudoresultativas (e consequentemente divergindo nas análises), mas parecem ser unânimes em apontar diferenças entre pseudoresultativas e resultativas.

No presente trabalho, o foco é abordar o efeito aspectual que a partícula agrega nas resultativas e pseudoresultativas. A próxima seção aborda essa questão.

\section{Aspecto nas complex particle constructions}

Com o objetivo de abordar a questão aspectual da partícula nas complex particle constructions, a primeira subseção retoma as construções (pseudo)resultativas e as analisa no tocante à aspectualidade, retomando as análises e generalizações da literatura. Em seguida, a próxima subseção adiciona as partículas às construções, na tentativa de esclarecer a função aspectual que a partícula tem na estrutura de evento.

\subsection{Aspecto nas (pseudo)resultativas}

Estruturas muito parecidas superficialmente com construções resultativas foram estudadas em português brasileiro (PB) pioneiramente por Foltran (1999) e Lobato (2004). A comparação com o PB, no contexto deste artigo, pode ser ilustrativa no sentido de que as estruturas nessa língua foram contrapostas às estruturas do inglês com base (entre outros elementos) em diferenças aspectuais. Vejamos alguns dados do PB:

(18) a. Ele cortou o cabelo curto.

b. Ele desenhou o círculo torto.

c. Ele fabricou a cadeira torta. ${ }^{12}$

d. Ela costurou a saia justa.

e. O engenheiro construiu a ponte sólida.

f. Deus criou os homens fracos. ${ }^{13}$ 
Os dados em (18), para Foltran (1999), são distintos das resultativas do inglês. A autora aponta como uma das bases de diferenciação a questão aspectual: em português brasileiro, o predicado secundário não tem a propriedade de transformar um evento não delimitado em delimitado, diferentemente das resultativas. Em PB, a adição do AP não altera a classe aspectual do verbo, e sua função (na linha argumentativa de Parsons) é a de modificação de um estado final.

Os dados em (18), portanto, estariam mais para pseudoresultativas. Neles, [V + DP] são eventualidades accomplishments, ou seja, [+télico]. Portanto, o predicado secundário não seria o responsável pela atribuição do telos por si só.

Vejamos agora as resultativas, exemplificadas em (15) e (16), acima. Os verbos são eventualidades do tipo atividade, ou seja, [-télico]. Seria contraditório dizer que a sequência [V + DP] forma um accomplishment, porque (i) não se pode generalizar o $\mathrm{DP}_{\mathrm{ACC}}$ como argumento do verbo (inclusive, a tendência analítica é não considerar $\mathrm{DP}_{\mathrm{ACC}}$ argumento de $\mathrm{V}$ ), e (ii) a supressão do sintagma resultativo gera dados agramaticais, portanto há que se considerar o AP para se caracterizar a classe aspectual. A conclusão mais plausível é tomar [V DP AP] nas resultativas como accomplishment, em que, composicionalmente, há um verbo de atividade cuja culminação, o telos, é formado pela adição do sintagma resultativo. Assume-se, portanto, que o resultado do evento, denotado no predicado secundário, representa a telicidade - uma vez que é a sua presença que caracteriza a eventualidade como accomplishment, uma atividade culminada, caracterizada por [+estágio] e [+télico].

Já a caracterização aspectual das pseudoresultativas, exemplificadas em (17), acima, pode ser mais intrincada. A primeira questão é se essas estruturas são as mesmas das apresentadas para o PB. Seguindo Knöpfle (2017), as pseudoresultativas apresentam subgrupos de construções: algumas têm equivalência estrutural entre PB e inglês (compare-se (17d) e (18a), e (17e) e (18d)), e outras não, sendo que o $\mathrm{PB}$ precisa de modificação extra no adjetivo para trazer a leitura de resultado (como em (17a-b-c). De toda a forma, se assumirmos que nas pseudoresultativas o predicado secundário modifica um estado final já denotado no conteúdo semântico [V DP], então não poderíamos dizer que esse predicado representa o telos da eventualidade.

\subsection{Aspecto nas complex particle constructions: resultativa + partícula}

De posse do exposto até agora, podemos finalmente observar as construções resultativas (e pseudoresultativas) em co-ocorrência com partículas (defendidas aqui como sendo de natureza aspectual).

Para as pseudoresultativas, Levinson (2007) realiza experimento que aponta para a grande produtividade de ocorrência desse tipo de construção juntamente com partículas. São exemplos da autora (p.159): 
(19) a. In ten minutes, Mary smoothed the ribbons out flat.

b. In fifteen minutes, Bill cooked the tomatoes up $d r y$.

c. In three minutes, Sarah grilled the steak up black.

Levinson $(2007,2010)$ analisa os adjetivos das pseudoresultativas como modificadores de um indivíduo criado, denotado na raiz lexical do verbo e sintaticamente ativo. Em Mary braided her hair tight, exemplo prototípico da autora, $\mathrm{o}$ adjetivo não modifica o objeto direto: o que se torna tight por meio do verbo braid não é o objeto direto the hair, mas sim the braid (a trança), criada pelo evento braiding (trançar).

Se de fato a eventualidade apresenta um resultado em $\left[\mathrm{V} \mathrm{DP}_{\mathrm{ACC}}\right]$, conforme defende a autora, e seguindo argumentação semelhante em Parsons (1990) e Foltran (1999), então o predicado secundário não adiciona telicidade ao evento, tampouco a partícula. O AP atua, dependendo da análise, como modificador ou de estado resultante ou indivíduo criado.

Resta a questão, portanto, da contribuição semântica da partícula. A proposta é que se trate de uma contribuição de natureza aspectual, que confere os sentidos de completude e/ou totalidade (com paráfrases para o PB tais como: completamente, totalmente).

Vejamos a construção resultativa em ocorrência juntamente com partículas.

(20) a. They painted the barn up red. (inglês)

b. Sie malten die Scheune rotan.(alemão)

Eles pintaram o celeiro vermelho PRT

'Eles pintaram e o celeiro ficou todo/completamente vermelho.'

Conforme exposto anteriormente, o predicado secundário da resultativa representa o telos da eventualidade. Uma outra possibilidade seria propor que o telos seja a combinação $\left[\mathrm{AP} \mathrm{DP}_{\mathrm{ACC}}\right.$ ]: se há uma ação verbal (representada no conteúdo lexical do verbo de atividade), e se o resultado dessa ação está denotado na combinação [ $\mathrm{AP} \mathrm{DP}_{\mathrm{ACC}}$ ], então essa própria combinação é a culminação do evento. Essa segunda possibilidade, inclusive, parece ser mais apropriada se adotarmos uma análise estrutural para resultativas, em que o $\mathrm{DP}_{\mathrm{ACC}}$ não é argumento do verbo, mas, sim, sujeito de uma SC complemento que tem o AP como predicado.

Em qualquer uma dessas duas possibilidades, a partícula não pode ser tomada como o telos do evento. Portanto, assim como para as pseudoresultativas, a proposta é que se trate de uma partícula de natureza aspectual que confere completude e/ou totalidade à eventualidade. ${ }^{14}$

Ainda referente às construções resultativas que ocorrem juntamente com partículas verbais, é digno de nota um último tema: trata-se da produtividade (e até mesmo da possibilidade) da co-ocorrência de construções resultativas com partículas verbais. 
Não é unanimidade na literatura a co-ocorrência de resultativas e partículas verbais. Algumas combinações são barradas por questões semânticas, outras sem uma razão semântica aparente (MÜLLER, 2002; NEELEMAN; VAN DE KOOT, 2002). Segue um exemplo em que há a restrição e um em que a co-ocorrência é possível:

(21) a. He cried his eyes red.(resultativa)

b. ${ }^{\star} \mathrm{He}$ cried his eyes out red. (partícula + resultativa)

(22) a. Daniel slept his way to the top.(resultativa)

b. Daniel slept his way up to the top.(resultativa + partícula)

Ainda assim, a exemplo de dados como em (20) e (22), partículas em construções resultativas são possíveis, e, quando ocorrem, a proposta é de que a partícula fornece uma contribuição aspectual no sentido de completude e/ou totalidade. Para (22), podemos ainda entender o sentido aspectual como sendo o de atingir o limite máximo em uma escala para cima. Vejamos mais exemplos de construções resultativas e partículas verbais, do alemão:

(23) a. Hans trank das Bierglas leeraus.

Hans bebeu o copo-de-cerveja vazio PRT

'Hans bebeu tudo do copo de cerveja, que ficou vazio.'

b. Maria hat den Teller leer aufgegessen. ${ }^{15}$

Maria teve o prato vazio PRT-comido.

'Maria comeu tudo do prato, deixando-o vazio.'

A princípio, em um contexto out of the blue, os dados em (23) podem ser interpretados como 'doppeltgemoppelt', i.e., expressão alemã usada para indicar que uma mesma coisa é dita duas vezes; no caso, se estaria dizendo duas vezes que 'o copo de cerveja' e 'o prato' ficaram vazios. Porém, dentro de um contexto específico, em que a redundância é necessária e intencional, os dados são aceitáveis. Nesse contexto, a informação aspectual da partícula é de reforçar a completude do evento.

Para finalizar a exemplificação da possibilidade de partículas em construções resultativas, seguem mais dois exemplos, autênticos, do inglês:

(24) Swept the floor up clean of all the rust that fell (...).

(Nastyz 28)

(25) Next we hammer the metal out flat again to the same thickness as the first time $(\ldots)$.

(Jones, 2010, p. 290) 
O limite da co-ocorrência verbo-partícula em construções resultativas é uma questão aberta que carece de mais estudo. Uma hipótese investigativa teria relação com a generalização empírica de que, na construção resultativa em línguas ocidentais germânicas, é possível a presença de apenas um sintagma resultativo. Então, se a partícula estiver se comportando como sintagma resultativo (e não partícula aspectual), a co-ocorrênciaseria barrada.

\section{Considerações Finais}

Este artigo abordou um subgrupo das complex particle constructions, notadamente as construções resultativas e pseudoresultativasque ocorrem juntamente com uma partícula verbal. O objetivo foi o de investigar a função da partícula na construção, argumentando-se que se trata de uma função de natureza aspectual, em que a partícula fornece um sentido de completude e/ou totalidade ao evento, ainda sendo possível que essa contribuição seja a de fornecer um limite escalar (para cima ou para baixo). Para tanto, o trabalho apresentou algumas noções básicas de aspecto a fim de esclarecer a terminologia e conceituação utilizadas. Em seguida, foram apresentadas as partículas que, segundo a literatura, apresentam características aspectuais, levantando-se a complexidade acerca da categorização gramatical das partículas verbais. $\mathrm{O}$ trabalho seguiu abordando as complex particle constructions segundo seu comportamento sintático, ou seja, construções que apresentam um predicado secundário/adicional (causativas, triádicas, objeto duplo e resultativas) juntamente com partículas. Para categorizar o subgrupo de construções, foco de investigação, foram apresentadas as construções resultativas e pseudoresultativas. Finalmente, a quarta seção apresentou as construções (pseudo)resultativas juntamente com partículas para prosseguir na abordagem aspectual da partícula verbal. Permanece em aberto a questão de certas restrições para a co-ocorrência da construção resultativa e a partícula verbal.

\section{Notas}

1. Opto aqui pela nomenclatura verbo-partícula como uma maneira de manter certa neutralidade quanto ao sentido composicional ou idiomático da construção, uma vez que o termo frasal verb parece carregar, a partir do contexto de inglês como segunda língua, uma nuance de idiomatização, haja vista as intermináveis listas de frasal verbs contidas nos livro didáticos.

2. Optou-se aqui por manter a terminologia original do inglês, seguindo den Dikken (1995).

3. Exemplos de Pierozan (2016, p.291, 292).

4. Exemplos de Endres (2013, p.40).

5. No decorrer da obra, den Dikken (1995) desenvolve a análise de partículas 'proper' enquanto preposições ergativas; diferentemente, preposições intransitivas seriam representantes inergativos da categoria P. Além de elementos da categoria P ergativos, as partículas não são atribuidoras de Caso.

6. A assunção de que o sintagma em itálico é 'adicional' ou secundário depende, obviamente, do tipo de descrição estrutural atribuída à estrutura. Nesse contexto, pode se entender como predicado secundário, grosso modo, um constituinte que 
participa de uma predicação em que há atribuição de uma propriedade a um nominal marcado tematicamente por um núcleo de um outro constituinte.

7. Vários autores, inclusive, argumentam que o $\mathrm{DP}_{\mathrm{ACC}}$ não é argumento de $\mathrm{V}$, mesmo em uma leitura de superfície em que o $\mathrm{DP}_{\mathrm{ACC}}$ possa ser entendido como objeto do verbo (as resultativas aparentemente transitivas). Um exemplo de análise nesses moldes é defendido por Hoesktra (1988, 1992), em que V tem como complemento uma SC que encerra uma relação de sujeito-predicado, sendo o sujeito o $\mathrm{DP}_{\mathrm{ACC}}$ de superfície e o predicado, o sintagma resultativo. Nesse sentido, o 'objeto afetado' pré-existe à ação verbal. O autor nota que verbos como 'paint' (em 'to paint a house') são ambíguos nesse sentido, por poderem tomar como complemento tanto um 'objeto afetado' quanto 'objeto alcançado'. Porém, em uma resultativa, tal ambiguidade não se coloca, uma vez que o DP 'the house' é 'objeto afetado' pela ação verbal. Knöpfle (2014) apresenta testes empíricos que embasam a anulação da leitura do $\mathrm{DP}_{\mathrm{ACC}}$ como argumento de $\mathrm{V}$ em resultativas aparentemente transitivas.

8. Se o $\mathrm{DP}_{\mathrm{ACC}}$ é objeto do verbo ou não depende da análise em questão, conforme colocado na nota anterior.

9. O inglês também apresenta ambiguidade para esse dado; parece que a leitura mais saliente é a intransitiva.

10. Resultativas com verbos inacusativos são também vistas na literatura; nesse caso, o sintagma resultativo predica do sujeito de superfície, originalmente gerado abaixo de V (ver LEVIN; RAPPAPORT, 1995; HOEKSTRA, 1992; KNÖPFLE, 2014).

11. Exemplos (a-b) de Geuder (2000, p. 69).

12. Exemplos de Foltran, (1999, p. 149-151).

13. Exemplos de Lobato (2004, p. 158,162,163).

14. Knöpfle (2018) propõe uma análise para resultativas em línguas ocidentais germânicas que contempla a partícula na construção, tomada como um núcleo Asp (de aspecto), nos moldes de den Dikken (1995), sem, no entanto, elaborar sobre a natureza (aspectual) da partícula em si.

15. Os equivalentes verbo-partícula em inglês seriam drink up e eat up.

\section{Referências}

BARBOSA, J. W. C. Variação paramétrica em predicados complexos e nomes compostos: um estudo translinguístico. 2012. Tese (Doutorado) - Universidade de São Paulo, São Paulo, 2012.

BOLINGER, Dwight. The phrasal verb in English. Cambridge, Mass.: Harvard University Press, 1971.

CAPPELLE, Bert. Particle patterns in English: a comprehensive coverage. 2005. Tese (Doutoradoem: de Taal- enLetterkunde: Germaanse Talen)- FaculteitLetteren, Katholieke Universiteit Leuven, Leuven, 2005.

CARRIER, J.; J. H. RANDALL. The Argument Structure and Syntactic Structure of Resultatives. Linguistic Inquiry 23(2), p. 173-234, 1992.

CHOMSKY, N. Barriers. Cambridge, Mass.: The MIT Press, 1986.

DIKKEN, M. Den. Particles: On the syntax of verb-particle, triadic and causative constructions. Oxford: Oxford UP, 1995.

DOWTY, D. Word meaning and Montague grammar: The semantics of verbs and times in generative semantics and in Montague's PTQ. Dordrecht: Reidel Publishing Company, 1979. 
EMONDS, J. Evidence that indirect object movement is a structure preserving rule. Foundations of Language, vol.8, p. 546-61, 1972.

EMONDS, J. A transformational approach to English syntax. New York: Academic Press, 1976.

EMONDS, J. A unified theory of syntactic categories. Dordrecht: Foris, 1985.

ENDRES, L. B. Semantic and syntactic issues on aspectual post-verbal particles. 2013. Dissertação (Mestrado) - Pontifícia Universidade Católica do Rio Grande do Sul, Porto Alegre, 2013.

FOLTRAN, M. J. G. D. As construções de predicação secundária no português do Brasil: aspectos sintáticos e semânticos. Tese (Doutorado) - Universidade de São Paulo, São Paulo, 1999.

FRASER, Bruce. The verb-particle combination in English. New York: Academic Press, 1976.

GEUDER, W. Oriented adverbs: Issues in the lexical semantics of event adverbs. 2000. Tese (Doutorado) - UniversitätTübingen, 2000.

HOEKSTRA, T. Small clause results.Língua,vol.74, p.101-139, 1988.

HOEKSTRA, T. Aspect and theta-theory. In: ROCA, I. M. (ed.). Thematic structure: Its role in grammar. Dordrecht: Foris, 1992. p. 145-174.

JONES, K. Rune Warriors: The Klendoran Chronicles Book One. Bloomington, IN: Author House, 2010.

KAYNE, R. Principles of particle constructions. In: GUÉRON, Jacqueline; OBENAUER, Hans-Georg.(eds.). Grammatical representation. Dordrecht: Foris, 1985. p.101-140.

KNÖPFLE, A. Resultativas em línguas ocidentais germânicas: generalizações descritivas, descobertas empíricas e questões analíticas. 2014. Tese (Doutorado) Universidade Federal do Paraná, Curitiba.

KNÖPFLE, A. Sobre resultativas e pseudoresultativas: distinções de base empírica. DELTA: Documentação de Estudos em Lingüistica Teórica e Aplicada, vol. 33, n. 2, p. 315-346, ago. 2017.

KNÖPFLE, A. Uma proposta de descrição estrutural para resultativas. Cadernos de Estudos Linguísticos, Campinas, v.60, n. 1, p. 90-126, jan./abr. 2018.

KRATZER, A. Building resultatives. In: MAIENBAUM, C.; WÖLLSTEIN-LEISEN, A. (eds.). Event arguments in syntax, semantics and discourse. Tübingen: Niemeyer, 2005. p. 177-212.

LAKOFF, George; JOHNSON, Mark. Metaphors we live by. Chicago: The University of Chicago Press, 2003. Primeira Edição, 1980.

LEVIN, B.; RAPPAPORT HOVAV, M. Unaccusativity: at the syntax-lexical semantics interface. (Linguistic Inquiry Monograph 26). Cambridge, Mass.: The MIT Press, 1995.

LEVINSON, L. The roots of verbs. Tese (Doutorado) - New York University, 2007.

LEVINSON, L. Arguments for pseudo-resultative predicates. Natural Language and Linguistic Theory, v. 28, n.1, p. 135-182, 2010.

LINDNER, Susan. A lexico-semantic analysis with verb-particle constructions with UP and DOWN. 1981.Tese (Doutorado) - Universidade da Califórnia, San Diego, 1981. 
LOBATO, L. Afinal, existe a construção resultativa em português? In: NEGRI, L.; FOLTRAN; M. J.; PIRES DE OLIVEIRA, R. (orgs.). Sentido e Significação. São Paulo: Contexto, 2004. p. 142-179.

MARCELINO, M. O Parâmetro de Composição e a Aquisição/Aprendizagem de L2. 2007. Tese (Doutorado) - Universidade Estadual de Campinas, Campinas, 2007.

MARCELINO, M. Resultativas em Português Brasileiro. Veredas, v. 18, n. 1, p. 121137, 2014.

MÜLLER, S. Complex predicates: Verbal complexes, resultative constructions, and particle verbs in German. Stanford: CSLI Publications, 2002.

NastyZ28 Forums. 2011. Disponível em: http://nastyz28.com/threads/burned-outon-88-s10-4x4-project.210355/). Acesso em: 10/09/2020.

NEELEMAN, A.; Van de KOOT, H. Bare resultatives. Journal of Comparative Germanic Linguistics, v.6, n. 1, p.1-52, 2002.

PARSONS, T. Events in the semantics of English. Cambridge, MA: MIT Press, 1990.

PIEROZAN, S. K. M. Motivação metafórica nos frasal verbs: uma concepção semântico-cognitiva. SCRIPTA, Belo Horizonte, v. 20, n. 40, p. 286-306, $2^{\circ}$ sem. 2016.

ROTHSTEIN, S. Structuring events: A study in the semantics of lexical aspect. Blackwell, Oxford, 2004.

RUDZKA-OSTYN, Brygida. Word power: Phrasal verbs and compounds. Berlin: Mouton de Gruyter, 2003.

WACHOWICZ, T. C.; FOLTRAN, M. J. G. D. Sobre a noção de aspecto. Cadernos de Estudos Linguísticos (UNICAMP), Campinas, v. 48, p. 211-232, 2007.

VENDLER, Z. Linguistics in philosophy. Ithaca, NY: Cornell University Press, 1967.

Recebido em: 30/04/2020 Aceito em: 03/08/2020 